\title{
Cumulative areawise testing in wavelet analysis and its application to geophysical time series
}

\author{
Justin A. Schulte \\ Department of Meteorology, Pennsylvania State University, University Park, Pennsylvania, USA \\ Correspondence to: Justin A. Schulte (jas6367@psu.edu)
}

Received: 14 May 2015 - Published in Nonlin. Processes Geophys. Discuss.: 29 July 2015

Revised: 12 January 2016 - Accepted: 3 February 2016 - Published: 1 March 2016

\begin{abstract}
Statistical significance testing in wavelet analysis was improved through the development of a cumulative areawise test. The test was developed to eliminate the selection of two significance levels that an existing geometric test requires for implementation. The selection of two significance levels was found to make the test sensitive to the chosen pointwise significance level, which may preclude further scientific investigation. A set of experiments determined that the cumulative areawise test has greater statistical power than the geometric test in most cases, especially when the signal-tonoise ratio is high. The number of false positives identified by the tests was found to be similar if the respective significance levels were set to 0.05 .
\end{abstract}

\section{Introduction}

In many research fields, it is of interest to understand the behavior of time series in order to achieve a deeper understanding of physical mechanisms or relationships. Such a task can be formidable given that time series are composed of oscillations, non-stationarities, and noise. A widely used method is wavelet analysis, which has proven useful in numerous geophysical investigations (Higuchi et al., 1999; Olsen et al., 2012; Meyers et al., 1993; Lee and Lwiza, 2008; Whitney, 2010; Wilson et al., 2014; Labat, 2008, 2010; Grinsted et al., 2004; Velasco and Mendoza, 2008; Schulte et al., 2016).

When using any time series extraction procedure it is important to assess the significance of the computed test statistic against some null hypothesis. In geophysical applications, for example, red noise is typically chosen as the null hypothesis. Torrence and Compo (1998) were the first to apply wavelet analysis in a statistical framework using point- wise significance testing, allowing deterministic features to be distinguished from stochastic features. In a pointwise significance test, one tests each estimated wavelet power coefficient against a stationary theoretical red-noise background spectrum. Despite the insights gained from the statistical procedure, Maraun and Kurths (2004) showed that it can lead to many spurious results simply due to multiple testing. Addressing the multiple-testing problem, Maraun et al. (2007) developed an areawise test that decides whether a pointwise significant result is a deterministic feature distinguishable from typical stochastic fluctuations by using basic properties of the continuous wavelet transform. A simpler procedure for addressing multiple testing problems is the geometric test developed by Schulte et al. (2015). The calculation of the critical level for the geometric test is much simpler than that for the areawise test because it is calculated using a basic Monte Carlo procedure that generates a null distribution of the test statistic.

Both the geometric and areawise tests suffer from a binary decision because one must choose both a pointwise significance level together with an areawise or geometric significance level. The problem with such a statistical construction is that the outcomes of the testing procedure may depend on the chosen pointwise significance level. For an ideal test, there is a single significance level that is chosen and the results of the testing procedure depend only on that significance level. Thus, the objectives of this paper are the following:

1. quantify how the binary decision of the geometric test can lead to ambiguity in interpreting results;

2. understand and quantify the evolution of pointwise significant regions under a changing pointwise significance level using persistent topology; 


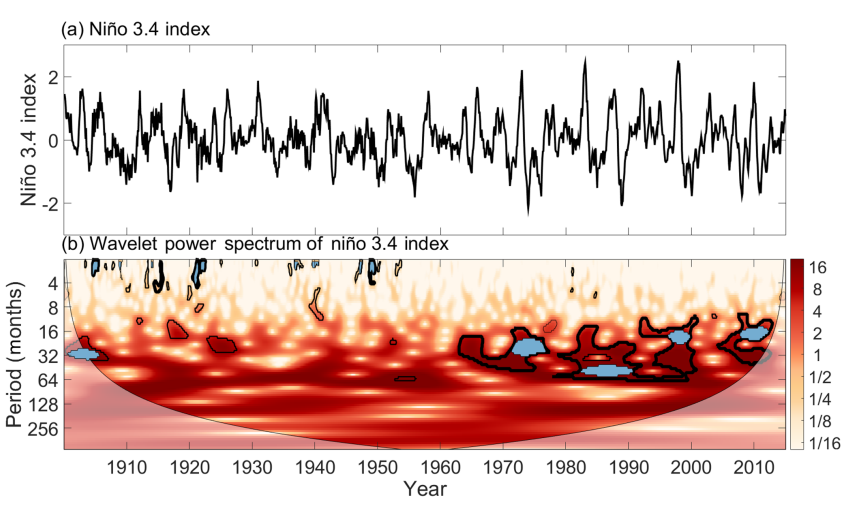

Figure 1. Wavelet power spectra of the Niño 3.4 index. Thin black contours enclose regions of $5 \%$ pointwise significance and thick contours indicate those patches that are geometrically significant at the $5 \%$ level. Light blue shading represents $5 \%$ areawise significant subsets of the patches. Light shading represents cone of influence, the region in which edge effects cannot be ignored.

3. design a statistical test, whose application only requires the choice of a single significance level.

Motivated by objectives 1 and 2, the approach to achieve objective 3 will be to consider the areas of pointwise significant regions over all pointwise significance levels, and hence the method will be called the cumulative areawise test.

The paper is organized as follows. The data used in applications of the significance tests are described in Sect. 2 and a brief description of wavelet analysis is provided in Sect. 3 . In Sect. 4, a review of existing statistical testing procedures is presented. The sensitivity of the geometric test to the chosen pointwise significance level is quantified in Sect. 5. The topological properties of red noise are analyzed in Sect. 6 and the cumulative areawise test is developed in Sect. 7. A comparison of the test in terms of statistical power to the existing geometric test is provided in Sect. 8. Applications of the test to prominent climate indices are presented in Sect. 9 and are followed by concluding remarks in Sect. 10.

\section{Data}

The Niño 3.4 index data from 1900 to 2014 were obtained from the National Center for Atmospheric Research. This index quantifies the strength of the El Niño-Southern Oscillation (ENSO) and is defined as sea surface temperature (SST) anomalies in the equatorial Pacific in the region bounded by $120-170^{\circ} \mathrm{W}$ and $5^{\circ} \mathrm{S}-5^{\circ} \mathrm{N}$ (Trenberth, 1997). The Pacific Decadal Oscillation index data were obtained from the University of Washington (http://research.jisao.washington. edu/pdo/PDO.latest) and describe detrended SST variability in the North Pacific poleward of $20^{\circ} \mathrm{N}$ latitude (Mantua and Hare, 2002).

\section{Wavelet analysis}

The wavelet transform of a time series $x_{n}(n=1, \ldots, N)$ with a wavelet function $\psi_{0}$ is given by

$W_{n}(s)=\sqrt{\frac{\delta t}{s}} \sum_{n^{\prime}=1}^{N} x_{n^{\prime}} \psi_{0}\left[\left(n^{\prime}-n\right) \frac{\delta t}{s}\right]$,

where $s$ is the wavelet scale, $\delta t$ is a time step determined by the data, and $N$ is the length of the time series. There are many kinds of wavelets, but perhaps the most common is the Morlet wavelet, the focus of this paper, which is given by

$\psi_{0}(\eta)=\pi^{-1 / 4} e^{i \omega_{0} \eta} e^{-\frac{1}{2} \eta^{2}}$,

where $\omega_{0}$ is the dimensionless frequency, $\eta=s \cdot t, t$ is time, and the wavelet scale is related to the Fourier period by $\lambda=$ $1.03 \mathrm{~s}$ if $\omega_{0}=6$. This particular wavelet balances both frequency and time localizations. Throughout the paper, $\omega_{0}=6$. The wavelet power spectrum is given by $\left|W_{n}(s)\right|^{2}$.

\section{Existing significance tests}

\subsection{Pointwise significance test}

Consider a first-order autoregressive (Markov) process

$x_{n}=\rho x_{n-1}+w_{n}$,

where $\rho$ is the lag- 1 autocorrelation coefficient, $w_{n}$ is Gaussian white noise, and $x_{0}=0$. The normalized theoretical power spectrum of the process is given by

$p_{f}=\frac{1-\rho^{2}}{1+\rho^{2}-2 \rho \cos (2 \pi f / N)}$,

where $f=0, \ldots, N / 2$ is the frequency index (Gilman et al., 1963). To obtain, for example, the $5 \%$ pointwise significance level ( $\alpha=0.05)$, one must multiply Eq. (4) by the 95th percentile of a chi-square distribution with 2 degrees of freedom and divide the result by 2 to remove the degreeof-freedom factor (Torrence and Compo, 1998). The result of the so-called pointwise testing procedure is a subset of wavelet power coefficients, whose values exceed the specified background noise spectrum. Such subsets will be referred to as patches.

\subsection{Areawise and geometric significance tests}

The areawise test developed by Maraun et al. (2007) takes advantage of how correlations between adjacent wavelet coefficients arising from the reproducing kernel produce patches that resemble the reproducing kernel. For patches generated from random fluctuations, the typical patch area is the area of the reproducing kernel. The areawise test assesses the significance of patches based on their area, where patches 


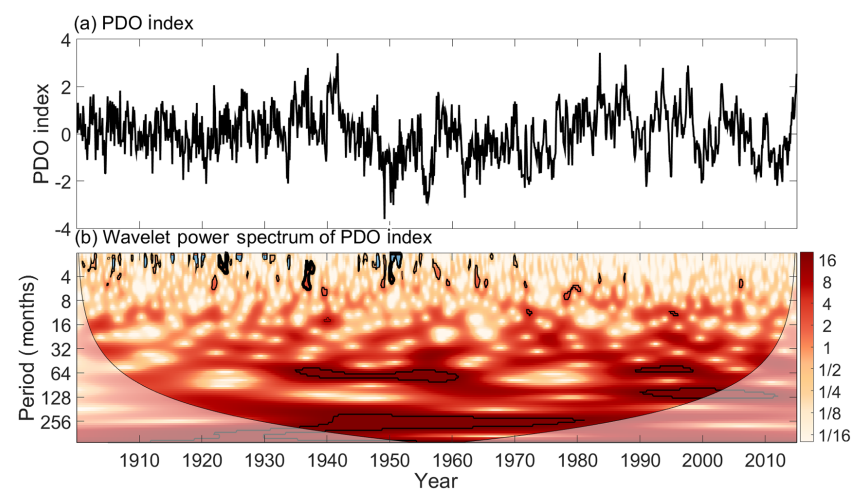

Figure 2. Same as Fig. 1 but for the PDO index.
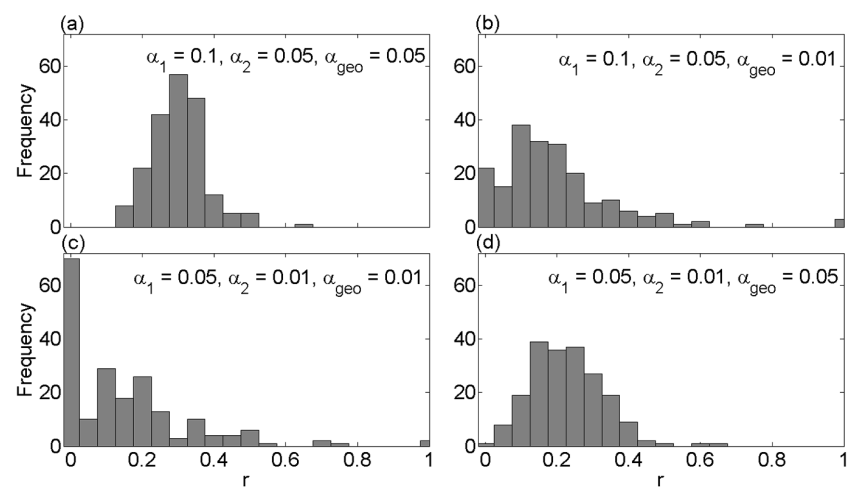

Figure 3. (a) A histogram of $r=\frac{N_{\alpha_{1}, \alpha_{2}}}{N_{\alpha_{1}}}$ for $\alpha_{1}=0.1, \alpha_{2}=0.05$, and $\alpha_{\text {geo }}=0.05$ obtained from the generation of 300 wavelet power spectra of red-noise processes of length 1000 with lag-1 autocorrelation coefficients equal to 0.5 . (b) Same as (a) but with $\alpha_{\text {geo }}=0.01$. (c) Same as (a) but with $\alpha_{1}=0.05, \alpha_{2}=0.01$, and $\alpha_{\text {geo }}=0.01$. (d) Same as (c) but with $\alpha_{\text {geo }}=0.05$.

with greater area are more statistically significant. The estimation of the critical level of the test involves a root-finding algorithm that is computationally inefficient. To remedy the computational drawback, Schulte et al. (2015) developed a geometric test that makes use of a normalized area. The normalized area allows patches at different scales to be compared simultaneously. The estimation of the critical level of the test is achieved simply through Monte Carlo methods by generating a large ensemble of patches under a null hypothesis to create a null distribution from which the critical level of the test can be obtained.

\subsection{Application of existing significance tests}

Shown in Fig. 2 is the wavelet power spectrum of the Niño 3.4 index. Large $5 \%$ patches were found and the largest was located in the time period 1950-2014 and in the period band 16-32 months. The large patches after 1950 were also found to be $5 \%$ geometrically significant (thick contours) and subsets of the patches were also found to be $5 \%$ areawise signif- icant (blue shading). Both the areawise and geometric tests identified few patches in the period band of 2 to 4 months as statistically significant. For the wavelet power spectrum of the Pacific Decadal Oscillation (PDO) index, a large patch centered at a period of 512 months extending from 1910 to 1980 was detected. Most of the patches, however, were located at periods less than 8 months, timescales not typically associated with the PDO. A few patches were identified as areawise and geometrically significant and such patches were in the 2 to 8 month period band.

\section{Sensitivity of the geometric test to the chosen pointwise significance level}

To show that the geometric test is sensitive to the chosen pointwise significance level, it will be useful to compute the quantity

$r=\frac{N_{\alpha_{1}, \alpha_{2}}}{N_{\alpha_{1}}}$.

The quantity $N_{\alpha_{1}, \alpha_{2}}$ is the number of geometrically significant patches at the pointwise significance level $\alpha_{1}$ that are also geometrically significant at the pointwise significance level $\alpha_{2} . N_{\alpha_{1}}$ is the number of patches at $\alpha_{1}$ that are geometrically significant at the level $\alpha_{\text {geo }}$. In the ideal situation, $r=1$, indicating that geometrically significant patches never lose their geometric significance as the pointwise significance level is increased. This case, however, is optimistic, as the calculation of geometric significance is rather stochastic.

To demonstrate the stochastic nature of the geometric test, $r$ was computed for 1000 wavelet power spectra of red-noise processes with lengths 1000 and $\rho=0.5$ under four scenarios. Scenario 1 is the case in which $\alpha_{1}=0.1, \alpha_{2}=0.05$, and $\alpha_{\text {geo }}=0.05$ (Fig. 3a). With the mean of $r$ (denoted by $\bar{r}$ hereafter) being 0.3 , it can hardly be expected for a geometrically significant patch at $\alpha_{1}=0.1$ to remain significant when the pointwise significance level is changed to $\alpha_{2}=0.05$, at least in the case of red-noise processes. Scenario 2, shown in Fig. 3 b, is the same as scenario 1 except that $\alpha_{\text {geo }}=0.01$. In this case, $\bar{r}=0.15$ suggesting that the geometric test is even more sensitive to the chosen pointwise significance level for smaller $\alpha_{\text {geo }}$.

In scenario 3, $\alpha_{1}=0.05$ and $\alpha_{2}=0.01$, with $\alpha_{\text {geo }}=0.01$. The distribution shown in Fig. $3 \mathrm{c}$ is even more skewed than that corresponding to scenario 2 , with $\bar{r}=0.05$. Also note that in many cases $r=0$, indicating that there are patches that are not geometrically significant for both $\alpha_{1}=0.05$ and $\alpha_{2}=0.01$. The reason is that some patches existed at $\alpha_{1}=$ 0.05 but did not exist at $\alpha_{2}=0.01$ so that their normalized areas are zero.

Scenario 4 is similar to scenario 3 except that $\alpha_{\mathrm{geo}}=0.05$. Although scenarios 3 and 4 used the same pointwise significance levels, the results differ, with $\bar{r}=0.22$. The results 
(a)

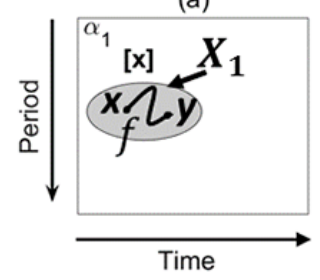

(e) (b)

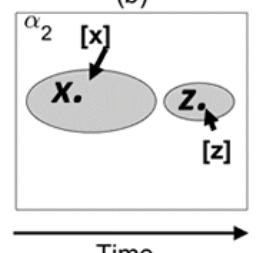

Time (c)

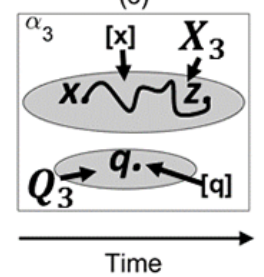

(d)

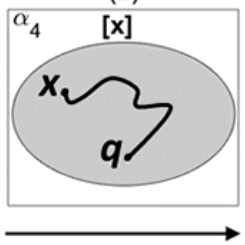

Time

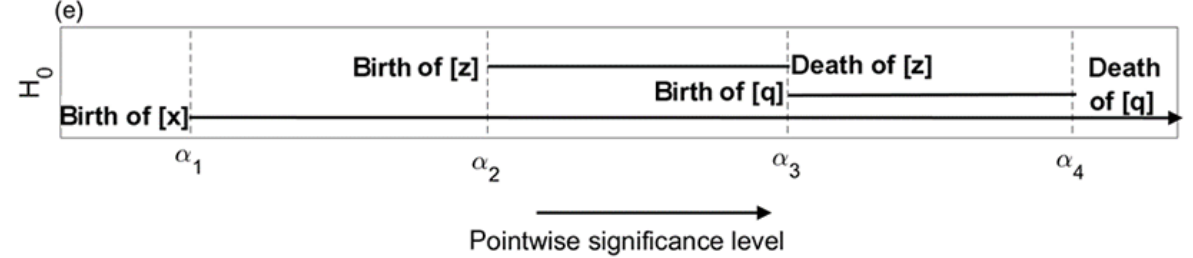

Figure 4. (a)-(d) The topological evolution of patches across four pointwise significance levels. (e) The barcode showing the birth and death of patches throughout the evolution process. Horizontal lines with arrows indicate those patches that never die.

are similar to that of scenarios 1 and 2, where increasing the pointwise significance level increased the sensitivity of the geometric test to the chosen pointwise significance level.

\section{Persistent topology}

\subsection{Persistent homology}

Before developing the cumulative areawise test, it will be necessary to understand the topology of features found in a typical wavelet power spectrum. It will be especially important to understand how the features evolve as the pointwise significance level is increased or decreased. Such information can be obtained using persistent homology, a tool in applied algebraic topology (Edelsbrunner and Harer, 2010). Persistent homology will provide a formal setting for quantifying the evolution of patches. Some formal definitions will be given below, but the reader is referred to Edelsbrunner and Harer (2010) for a more detailed description of persistent homology.

A pointwise significance patch will be defined formally as follows. A path in a set $X$ is defined as a continuous function $f:[01] \rightarrow X$ (Lipschutz, 1965). A set $X$ is said to be path connected if any two points $x$ and $y$ in $X$ can be joined by a path. The path component of a set $X$ is the maximal path-connected subset of a set. Intuitively, one can think of a path component as an isolated piece of the set. In the present setting, patches are path-connected components because they represent isolated pieces of the set consisting of all wavelet power coefficients that are pointwise significant.

Denote by $P$ the set of all pointwise significant wavelet power coefficients that are significant at the $\alpha$ level. Then two points $x, y \in P$ will be called homologous (written $x \sim y$ ) at $\alpha$ if there exists a path $f:[01] \rightarrow P$ such that $f(0)=x$ and $f(1)=y$ (Fig. 4a). The definition implies that two points are homologous when they can be joined by a continuous path. The set of all points that are homologous to $x$ form an equivalence class called a homology class that is denoted by

$[x]=\{y \in P: y \sim x\}$.

The set of all homology classes of $P$ will be denoted by $H_{0}(P)$, where $H_{0}(P)$ is called the zero-dimensional homology group (Hatcher, 2002). Each member of a homologous class is homologous but no two points from distinct homology classes are homologous. The homology classes form a partition of $P$ into path-connected components and therefore patches at a given pointwise significance level can be regarded formally as homology classes. Mathematically, we have the quotient

$H_{0}(P)=P / \sim=\{[x]: x \in P\}$

and the fundamental theorem of equivalence classes (Lipschutz, 1965) says that $H_{0}(P)$ forms a partition of $P$.

The number of equivalence classes, $\beta_{0}$, can change as $\alpha$ is increased or decreased. A homology class at $\alpha_{2}$ will be said to be born at $\alpha_{2}$ if it did not exists at $\alpha_{1}$, for every $\alpha_{1}<\alpha_{2}$. The homology class $[z]$ shown in Fig. $4 \mathrm{~b}$, for example, was born at $\alpha_{2}$. Suppose that a homology class [x] is born at $\alpha_{1}$ and $[y]$ is born at $\alpha_{2}$ for $\alpha_{1}<\alpha_{2}$. Then $[x]$ will be said to be older than $[y]$.

Homology classes can also die. The death of a homology class will simply mean that two classes have merged so that two points that are not homologous at $\alpha_{1}$ become homologous at $\alpha_{2}$. To see this, consider the homology classes $[x]$ and $[z]$ at $\alpha_{2}$ shown in Fig. 4b. They both represent different homology classes because the point $x$ cannot be connected to $z$ by a path. At $\alpha_{3}$, on the other hand, $x \sim z$ or $z \sim x$ so that $x$ is a member of $[z]$ or $z$ is a member of $[x]$. The result is a reduction in the number of homology classes. When homology classes merge, it will be necessary to use the Elder rule (Edelsbrunner and Harer, 2010) from persistent homology to 
determine which classes die from a merger and which ones live. The Elder rule states that when two classes merge, the older class will continue to live. Therefore, according to the Elder rule, the class $[x]$ will live after the merger with the class $[z]$ at $\alpha_{3}$ and $[z]$ will die. The reason $[x]$ lives is because it was born at $\alpha_{1}$ and [z] born was born at $\alpha_{2}$ so that $[x]$ is older. The lifetime or persistence index of a homology class will be defined as the difference between the pointwise significance level at which it dies and the one at which it was born. If a homology class never dies, then its persistence index, by convention, will be set to infinity.

The evolution of a homology class can be monitored using a barcode (Ghrist, 2008), which is a collection of horizontal lines representing the birth and death of homology classes. Following the convention of persistent homology, the $y$ axes of barcodes will be denoted by $H_{0}$ and the $x$ axes will be the pointwise significance level. In the barcode, the birth of a homology class will begin a horizontal line segment at the pointwise significance level at which it was born. The line segment will terminate at the pointwise significance level at which it dies.

An example barcode is shown in Fig. 4e for the evolution of homology classes shown in Fig. 4a-d. The homology class $[x]$ was born at $\alpha_{1}$ so that a horizontal line begins at $\alpha_{1}$. The patch does not merge with another patch at $\alpha_{2}$ so that the horizontal line continues through $\alpha_{2}$. The homology class [z] is born at $\alpha_{2}$ and the birth of the homology class results in a new line starting from $\alpha_{2}$. The merger of the homology classes $[x]$ and $[z]$ at $\alpha_{2}$ results in the death of [z]. According to the Elder rule, the horizontal line corresponding to $[x]$ in the barcode continues through $\alpha_{3}$, but the line corresponding to $[z]$ terminates at $\alpha_{3}$. Also note the birth of a new homology class $[q]$ at $\alpha_{3}$ and the corresponding beginning of the line segment. Another merger occurs at $\alpha_{4}$ because $x \sim q$ and the Elder rule determines that the line segment for $[q]$ ends and the horizontal line for $[x]$ continues. The arrow indicates that $[x]$ never dies.

\subsection{Persistent homology of red noise}

To understand the topology of patches generated from rednoise processes, it is useful to use Monte Carlo methods to determine the number of patches at a particular pointwise significance level. Shown in Fig. 5 is the ensemble mean of the number of patches as a function of $\alpha$. The curve was obtained by generating 100 wavelet power spectra of rednoise processes of length 300 and computing $\beta_{0}$ for each of the wavelet power spectra at each pointwise significance level. The number of patches reached minima at $\alpha=0.01$ and $\alpha=0.99$ and a maximum at $\alpha=0.18$.

To understand more fully the curve shown in Fig. 5, the persistent homology of patches generated from red-noise processes of length 150 was computed as $\alpha$ varied from 0.01 to 0.99 . Barcodes representing the evolution of patches (homology classes) in the wavelet power spectra were also com-

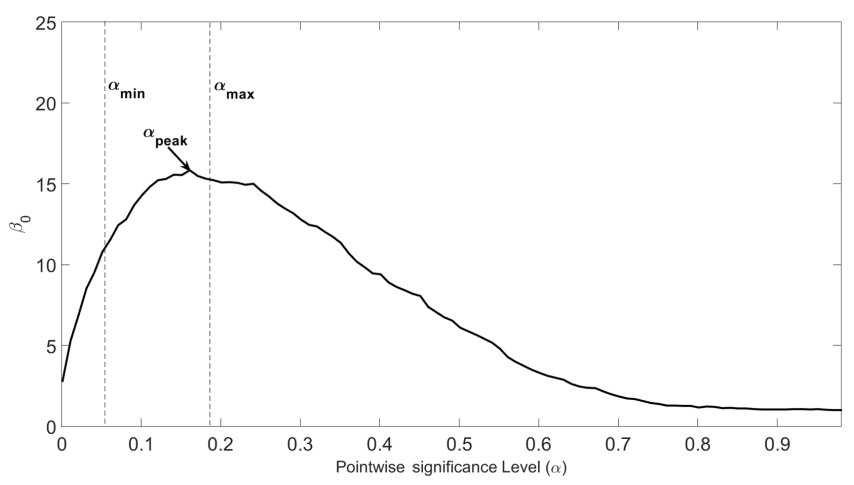

Figure 5. The mean number of patches as a function of $\alpha$. The curve was obtained by generating 100 wavelet power spectra of red-noise processes of length 300 and computing $\beta_{0}$ for each of the wavelet power spectra at each pointwise significance level. The quantities $\alpha_{\min }$ and $\alpha_{\max }$ are the lower and upper bounds of the computation interval for the cumulative areawise test and $\alpha_{\text {peak }}$ is the pointwise significance level for which the number of patches reaches a maximum within the computation interval.

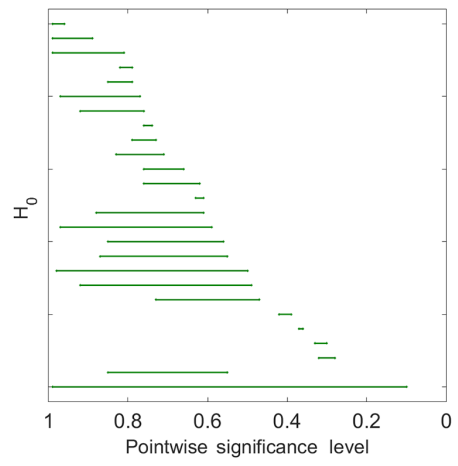

Figure 6. Example barcode for $H_{0}$ corresponding to a wavelet power spectrum of a red-noise process with length 150 and $\rho=0.5$.

puted. In each case, $\rho=0.5$, but the results are identical for other autocorrelation coefficients. Shown in Fig. 6 is a barcode corresponding to a typical wavelet power spectrum of a red-noise process. Recalling that the beginning of the line segment represents the birth of patches, the barcode indicates that a few patches were born at $\alpha=0.02$. As $\alpha$ increases to $\alpha=0.3$ more patches are born, consistent with how more spurious results occur for larger pointwise significance levels. Note that, for $\alpha>0.2$, patches begin to die, representing the merger of smaller patches into larger patches. The merging process occurs until $\alpha=0.7$, at which point all patches have merged into a single patch. To show that the distribution of persistence indices for patches generated from red-noise processes is not random, 100 wavelet power spectra of rednoise processes were generated and the persistence indices for all patches in each wavelet power spectrum were computed (Fig. 7). The resulting distribution indicates that persistence indices are typically 0.01 and relatively few patches 


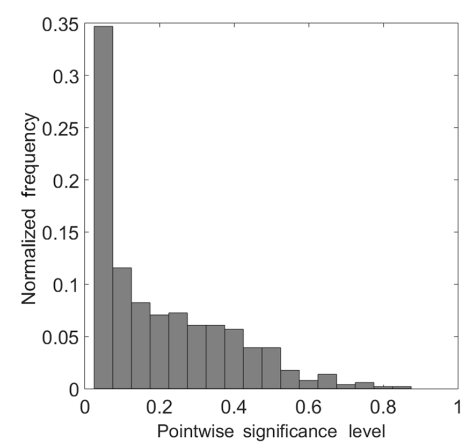

Figure 7. Distribution of persistence indices representing the lifetimes of patches. The distribution was obtained by generating 1000 wavelet power spectra of red-noise processes with lengths 500 and $\rho=0.5$. Persistence indices equal to infinity are excluded from the distribution.

live longer than 0.6. Overall, the distribution characterizes patches generated from red-noise processes as short lived.

\section{Development of the cumulative areawise test}

\subsection{Geometric pathways}

The first step of the cumulative areawise test is to define the geometric evolution of a patch across a finite set of pointwise significance levels. The notion of evolution will be made precise by introducing the concept of a geometric pathway, which is defined as a collection $\mathcal{P}$ of $L$ patches at the corresponding pointwise significance levels $\alpha_{1}<\alpha_{2}<\ldots<\alpha_{L}$ such that

$P_{1} \subset P_{2} \subset P_{3} \subset \ldots \subset P_{L}$

and

$g_{1}<g_{2}<g_{3} \ldots<g_{L}$,

where each $g_{j}$ is a normalized area corresponding to the patch $P_{j}$. For this testing procedure, the normalized area will be calculated by dividing the patch area by the scale coordinate of the centroid squared. The inequalities (Eq. 9) are guaranteed to hold for any nested sequence (Eq. 8) (Appendix A). The length of a pathway will be given by $L$ the number of elements in the pathway. The interval $I=$ $\left[\alpha_{\min }, \alpha_{\max }\right]$ will be called the computation interval and the discrete spacing between adjacent pointwise significance levels, $\Delta \alpha$, will be referred to as the resolution.

There is a close relationship between geometric pathways and persistent homology. The birth of homology classes also signifies the creation of a geometric pathway. In contrast, the death of homology classes does not indicate the termination of a geometric pathway. According to Eq. (9), once the first element of the pathway is created the pathway cannot terminate because elements grow relative to the first element.
The number of geometric pathways that are computed in a given wavelet power spectrum is related to $\Delta \alpha$ and the persistent homology of patches quantified in Sect. 6.2. To see this, suppose geometric pathways were calculated at the resolution $\Delta \alpha=\alpha_{3}-\alpha_{1}$ starting at $\alpha_{1}=\alpha_{\min }$ and ending at $\alpha_{3}=\alpha_{\max }$ as shown in Fig. 4. At this resolution, two pathways would be created, $X_{1} \subset X_{3}$ and $Q_{3}$. If the point $z$ had not become homologous to the point $x$ at $\alpha_{3}$, then an additional pathway corresponding to $[z]$ would have been calculated because it would still be a path-connected component (i.e., a patch) distinct from $X_{3}$ and $Q_{3}$. The argument suggests that only geometric pathways comprised of patches with lifetimes greater than or equal to $\Delta \alpha$ will be detected. A natural questions thus arises: how small should be $\Delta \alpha$ ? It should certainly be made small enough to adequately capture the birth and merging of patches. The distribution of persistent indices shown in Fig. 7 suggests that $\Delta \alpha=0.01$ because most persistent indices are at that value. However, the discussion in Sect. 8 will suggest a coarser resolution may be used without altering the statistical properties of the test.

\subsection{Test construction}

One can associate to each geometric pathway a test statistic, which will be the total sum of normalized areas

$\gamma=\sum_{j=1}^{L} g_{j}$

The calculation of the critical level for the test can be computed using Monte Carlo methods by first fixing $I$ and $\Delta \alpha$. Secondly, one generates red-noise processes with the same autocorrelation coefficients as the input time series and calculates synthetic wavelet power spectra corresponding to each red-noise process. The final step is to compute $\gamma$ for every pathway. The calculation results in a null distribution from which the desired critical level of the test can be obtained. The critical level corresponding to the $5 \%$ significance level of the test, as an example, is the 95th percentile of the null distribution.

A null distribution calculated for a red-noise process with $\rho=0.5$ is shown in Fig. 8. In the experiments, $\alpha_{\min }=0.02$ and $\alpha_{\max }=0.82$ with $\Delta \alpha=0.02$. The distribution of $\gamma$ is generally similar to the shape of the distribution for the persistence indices for $H_{0}$ (Fig. 7), where the smallest values of $\gamma$ are preferred. It turns out that the distribution of $\gamma$ can be well described by an exponential distribution. Using the method of maximum likelihood (Weerahandi, 2003), a theoretical exponential distribution was fitted to the empirical distribution, where the empirical distribution was found to be best described by an exponential distribution with mean 6.5. To show that the theoretical distribution models the empirical distribution, the percentiles of a theoretical exponential distribution with mean 6.5 were plotted as a function of the percentiles of the empirical distribution (Fig. 8b). The linear 

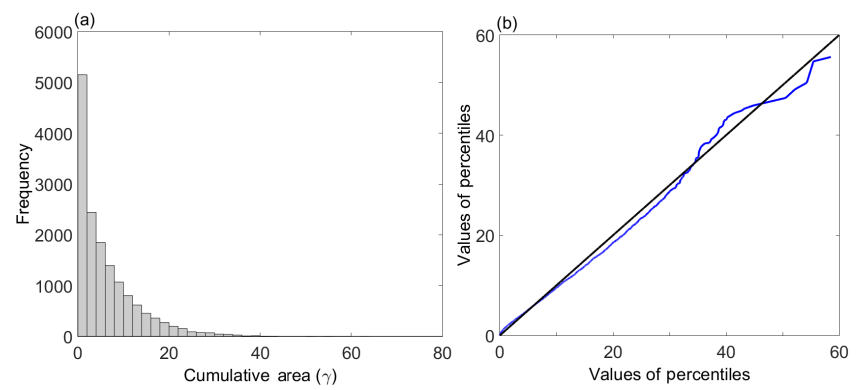

Figure 8. (a) Null distribution of $\gamma$ obtained by generating 10000 geometric pathways under the null hypothesis of red-noise, where the red-noise processes were of length 1000 and had lag-1 autocorrelation coefficients equal to 0.5. (b) Percentiles of a theoretical exponential distribution with mean 6.5 plotted as a function of the percentiles calculated from the distribution shown in (a).

relationship between the percentiles shown in Fig. 8b indicates that the theoretical distribution well models the empirical distribution, with the 95th percentiles only differing by 1.0 .

Associated with each element of the geometric pathway is the quantity

$\gamma_{j}=\sum_{i=0}^{L-j} g_{L-i}$

which represents the cumulative sum of the last $L-j+1 \mathrm{el}-$ ements of the pathway. One can calculate a $p$ value for every pathway element using Eq. (11) by comparing each $\gamma_{j}$ to the null distribution. Mathematically, for a null distribution $\gamma_{\text {null }}$ the $p$ values are given by

$p_{j}=\operatorname{Pr}\left(\gamma_{\mathrm{null}} \geq \gamma_{j}\right)$.

The pathway element $P_{j}$ will be said to be cumulative areawise significant at the $\alpha_{\mathrm{c}}$ significance level if $p_{j}<\alpha_{\mathrm{c}}$. The union of all cumulative areawise significant pathway elements will be the output of the testing procedure.

The $p_{j}$ satisfy

$p_{1}<p_{2}<p_{3} \ldots<p_{L}$

because $\gamma_{i}>\gamma_{j}$ for $i>j$. The inequality (Eq. 13) and nested sequence (Eq. 8) together show that the cumulative areawise significance of wavelet power coefficient is a monotonic function of the pointwise significance. To see this, denote by $x_{j}$ a wavelet power coefficient of the patch $P_{j}$ in a geometric pathway. If $p_{j}^{\mathrm{pw}}$ is the $p$ value of $x_{j}$ associated with the pointwise test, then $p_{i}^{\mathrm{pw}}>p_{j}^{\mathrm{pw}}$ for $i>j$. Let $F$ be a function assigning to every $p_{j}^{\mathrm{pw}}$ a $p_{j}$. The function $F$ is everywhere monotonically increasing because $p_{i}^{\mathrm{pw}}>p_{j}^{\mathrm{pw}}$ implies that $F\left(p_{i}^{\mathrm{pw}}\right)=p_{i}>p_{j}=F\left(p_{j}^{\mathrm{pw}}\right)$ for $i>j$ by inequality (Eq. 13). This monotonicity property is not shared

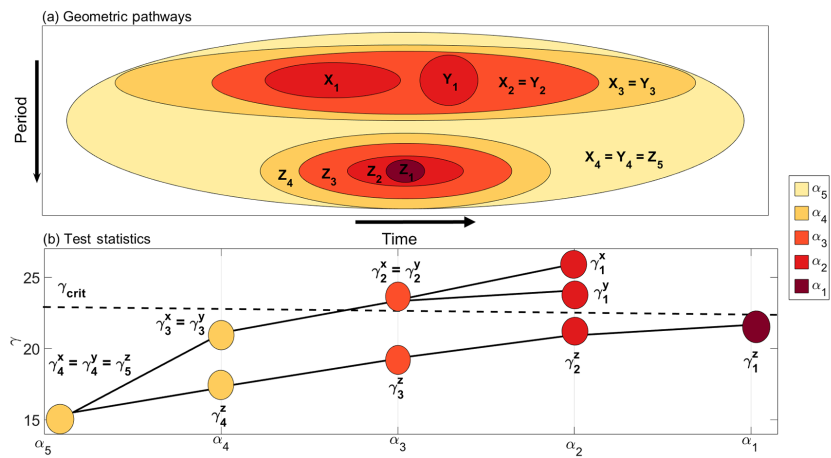

Figure 9. (a) Geometric pathway of three significance patches, $X$, $Y$, and $Z$ in the interval $I=\left[\alpha_{1}, \alpha_{5}\right]$. (b) The cumulative areas of geometric pathway elements, where the summation begins at $\alpha_{5}$, and the dotted line represents the critical level of the cumulative areawise test.

by the areawise or geometric tests, where there is no oneto-one function between the pointwise significance $p$ values and $p$ values for the areawise or geometric tests. In other words, wavelet power coefficients of different pointwise significance can have identical areawise or geometric significance. The monotonicity property also implies that each $p_{j}$ is only a function of $p_{j}^{\mathrm{pw}}$ and thus it has been shown that the cumulative areawise test is free of a binary decision (objective 3).

\subsection{Application to ideal pathways}

To illustrate the testing procedure, it is perhaps best to consider an ideal case (Fig. 9). Consider the pathway $X$, which can be written explicitly as

$X_{1} \subset X_{2} \subset X_{3} \subset X_{4}$.

The patch exists at $\alpha_{1}^{x}=\alpha_{2}, \alpha_{2}^{x}=\alpha_{3}, \alpha_{3}^{x}=\alpha_{4}$, and $\alpha_{4}^{x}=$ $\alpha_{5}=\alpha_{\max }$. The test statistics, using Eq. (11), for the geometric pathway are

$\gamma_{1}^{x}=g_{1}^{x}+g_{2}^{x}+g_{3}^{x}+g_{4}^{x}$,

$\gamma_{2}^{x}=g_{2}^{x}+g_{3}^{x}+g_{4}^{x}$,

$\gamma_{3}^{x}=g_{3}^{x}+g_{4}^{x}$,

and

$\gamma_{4}^{x}=g_{4}^{x}$,

where $g_{j}^{x}$ denotes the normalized area of a pathway element at $\alpha_{j}^{x}$. According to Fig. $9 \mathrm{~b}$, both $X_{1}$ and $X_{2}$ are cumulative areawise pathway elements because $\gamma_{1}^{x}, \gamma_{2}^{x}>\gamma_{\text {crit }}$. The output of the testing procedure is therefore given by

$X_{\text {sig }}=X_{2}=X_{1} \cup X_{2}$

A similar results holds for the pathway $Y$, where the output of the testing procedure is

$Y_{\text {sig }}=X_{2}=Y_{1} \cup Y_{2}$. 
The pathway $Z$ shown in Fig. 9a can be written as

$Z_{1} \subset Z_{2} \subset Z_{3} \subset Z_{4} \subset Z_{5}$.

The test statistics associated with each of the five pathway elements are

$\gamma_{1}^{z}=g_{1}^{z}+g_{2}^{z}+g_{3}^{z}+g_{4}^{z}+g_{5}^{z}$,

$\gamma_{2}^{z}=g_{2}^{z}+g_{3}^{z}+g_{4}^{z}+g_{5}^{z}$,

$\gamma_{3}^{z}=g_{3}^{z}+g_{4}^{z}+g_{5}^{z}$,

$\gamma_{4}^{z}=g_{4}^{z}+g_{5}^{z}$,

and

$\gamma_{5}^{z}=g_{5}^{z}$

As shown in Fig. 9b, none of the test statistics exceed $\gamma_{\text {crit }}$ and therefore the pathway elements are not cumulative areawise significant. The total output of the testing procedure in this case will be

$X_{2}=X_{\text {sig }} \cup Y_{\text {sig }}$

\section{Comparison with the geometric test}

\subsection{True positive detection}

With the cumulative areawise test now developed, it will be useful to assess the statistical power of the test relative to that of the geometric test. The first aspect of the assessment will be to quantify how well both tests detect true positive results. To do so, let

$x(t)=A \sin (2 \pi f t)+w(t)$

be a sinusoid with amplitude $A$, frequency $f$, and additive Gaussian white noise $w(t)$. The goal will be to evaluate the ability of both tests to detect true positives within a particular period band. A theoretical patch to which the ability of the geometric and cumulative areawise tests were compared was constructed as follows: (1) the time series $x(t)$ for all $t \in[0,500]$ was generated but with no additive white noise, (2) the wavelet power spectrum of $x(t)$ was computed and the $5 \%$ pointwise significance test was performed on the wavelet power spectrum, and (3) the width of the significance patch in the wavelet power spectrum was calculated at $t=250$ where edge effects are negligible. The theoretical patch is indicated by dotted lines in Fig. 10, where the theoretical patch is a rectangle of fixed width extending from $t=0$ to $t=500$. In all experiments, $\alpha_{\max }=0.18$ and $\Delta \alpha=0.02$, but implications of other choices are discussed at the end of the section.

Let $P_{\text {geo }}$ be the union of all pointwise significance patches at $\alpha$ that are geometrically significant at the $\alpha_{\text {geo }}$ level and let $P_{\text {theory }}$ be the theoretical patch. Then

$r_{\mathrm{a}}=\frac{A_{P_{\text {geo }} \cap P_{\text {theory }}}}{A_{P_{\text {theory }}}}$

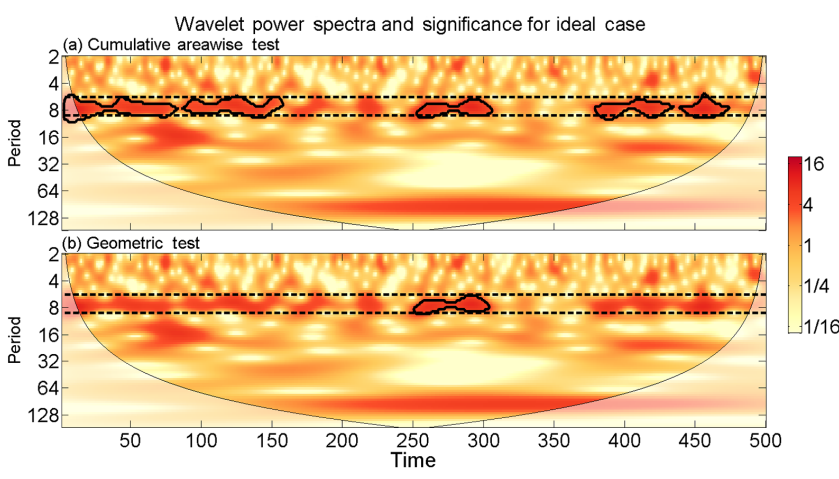

Figure 10. (a) Cumulative areawise test applied to a sinusoid with a frequency of 0.8 and amplitude equal to 0.8 . Signal-to-noise ratio is 1.0. Contours represent patches that are elements of $5 \%$ significant pathways. Dotted lines represent the upper and lower boundaries of a theoretical patch obtained by generating the wavelet power spectrum of a pure sine wave and calculating the width of the patch at $t=250$. (b) Same as (a) except for the geometric test with $\alpha=0.05$ and $\alpha_{\text {geo }}=0.05$. Contours represent patches that are geometrically significant.

represents the areal fraction of $P_{\text {theory }}$ detected by the geometric test. In Eq. (29), $A_{P_{\text {geo }} \cap P_{\text {theory }}}$ denotes the area of $P_{\text {geo }} \cap P_{\text {theory }}$ and $A_{P_{\text {theory }}}$ denotes the area of $P_{\text {theory }}$. If $r_{\mathrm{a}}=1$, then the test detected all of the true positive results that are known by construction. Small values of $r_{\mathrm{a}}$ indicate that the tests performed poorly, detecting only a fraction of the theoretical patch to be significant. A similar construction can be made for the cumulative areawise test by replacing $P_{\text {geo }}$ with $P_{\mathrm{c}}$. Figure 10a illustrates the procedure for the cumulative areawise test when $f=0.8, A=1.0$, and the signalto-noise ratio (defined below) $\sigma=1.0$. As indicated by the thick black contours, the cumulative areawise test was able to detect $30 \%$ of the true positives comprising the theoretical patch, whereas Fig. 10b shows that the geometric test was only able to detect $10 \%$ of the true positives. It will be necessary to compute $N=1000$ values of $r_{\mathrm{a}}$ for different values of $f$ and $\sigma$ to determine if the tests truly perform differently. The signal-to-noise ratio is defined as

$\sigma=10 \log \left(\frac{p_{\text {signal }}}{p_{\text {noise }}}\right)$,

where

$p_{\text {signal }}=\frac{A^{2}}{2}$,

$p_{\text {noise }}$ is the average power of the Gaussian white noise, and $\sigma$ is measured in decibels (dB). It is also noted that because $\sigma$ and $A$ do not vary independently there is no need to perform different experiments for different values of $A$. For the experiments, $A$ was set to 1.0 .

In the first experiment, the cumulative areawise significance level (denoted by $\alpha_{\mathrm{c}}$, hereafter) was set to $0.05, \alpha_{\mathrm{geo}}=$ 
0.05 , and $\alpha=0.01,0.05,0.1$. The value of $\sigma$ was varied from 5 to $5 \mathrm{~dB}$. The results are shown in Fig. 11a. For both tests, the ability to detect true positives increased with increasing signal-to-noise level. At low signal-to-noise ratios, the tests performed similarly, detecting on average $10 \%$ of true positives. Differences between the test performances became larger as $\sigma$ was increased and the cumulative areawise test outperformed the geometric test regardless of the chosen pointwise significance levels when $\sigma \geq-2.5 \mathrm{~dB}$. A second experiment was conducted using $\alpha_{\mathrm{c}}=0.01$ and $\alpha_{\text {geo }}=0.01$ (Fig. 11b). The results were found to be similar to that of the first experiment except that $r_{\mathrm{a}}$ was generally smaller for both tests. The result is consistent with how the significance levels of the tests were increased. The results indicate that the cumulative areawise test is particularly useful in low-noise situations but one can expect the test to detect more true positives even in high-noise conditions. In agreement with Fig. 3, the performance of the geometric test depended strongly on the chosen pointwise significance level, especially when the signal power was high.

Additional experiments were performed using different values of $f$. True positive detection, for a fixed $\sigma$, was generally found to increase for larger $f$, though the cumulative areawise test was still found to detect more true positives. Additionally, it was found that true positive detection changed little if $\Delta \alpha$ was set to a value less than 0.02. Setting $\Delta \alpha$ to be greater than 0.03 was generally found to result in a decrease in true positive detection. On the other hand, true positive detection increased dramatically as $\alpha_{\max }$ increased but with the caveat that the areas of spurious patches found outside the theoretical patch were larger.

\subsection{False positive detection}

The false positive detection of both tests depends on the topology of patches. The number of false positives produced by the geometric test performed at the pointwise significance level $\alpha$ on average will be

$N_{\text {geo }}\left(\alpha, \alpha_{\text {geo }}\right)=\alpha_{\text {geo }} \beta_{0}(\alpha)$.

For the cumulative areawise test, the number of false positives produced will be on average

$N_{\mathrm{c}}\left(\alpha_{\text {peak }}, \alpha_{\mathrm{c}}\right)=\alpha_{\mathrm{c}} \beta_{0}\left(\alpha_{\text {peak }}\right)$,

where $\alpha_{\text {peak }}$ satisfies $\alpha_{\text {min }} \leq \alpha_{\text {peak }} \leq \alpha_{\text {max }}$ and denotes the pointwise significance level for which $\beta_{0}$ locally reaches a maximum (Fig. 5). If $\alpha_{\mathrm{c}}=\alpha_{\mathrm{geo}}$, then the ratio of false positives for both tests is

$r_{\text {false }}=\frac{\beta_{0}(\alpha)}{\beta_{0}\left(\alpha_{\text {peak }}\right)}$.

Thus, if $\alpha=\alpha_{\text {peak }}$ both tests on average will have the same number of false positive results. On the other hand, $r_{\text {false }}<1$ if $\alpha_{\max }<\alpha<0.18$. According to Fig. $5, N_{\text {geo }}(0.05,0.05)$ is
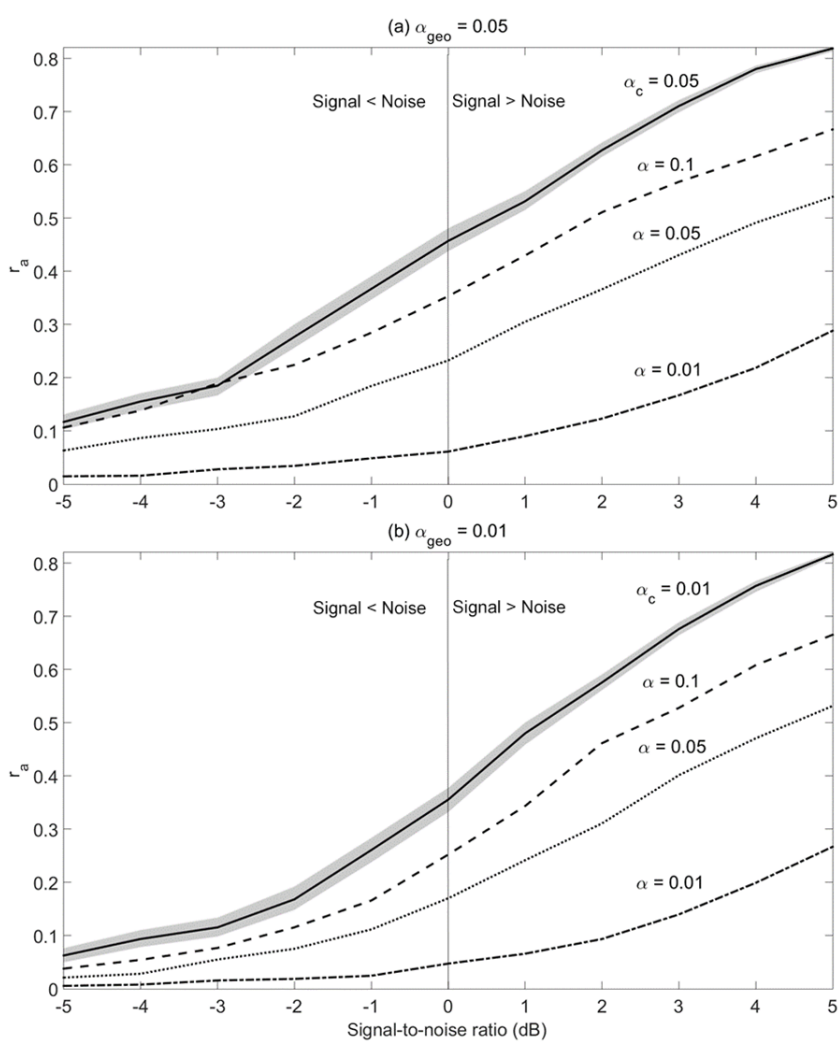

Figure 11. (a) The ensemble mean $r_{\mathrm{a}}$ as a function of the signalto-noise to ratio for the areawise test with $\alpha_{\mathrm{c}}=0.05$ and the geometric test with $\alpha_{\text {geo }}=0.05$. Gray shading represents the $95 \%$ confidence interval and all means for the geometric test are significantly different at the $5 \%$ level from the means for the areawise test except for those corresponding to the $\alpha=0.01$ curve for signal-tonoise ratios less than -1.5 . The confidence intervals and statistical significance were obtained by the bootstrap method (Efron, 1979). The data for each signal-to-noise-ratio were sampled with replacement 1000 times to generate a distribution of bootstrap replicates, from which $95 \%$ confidence intervals were obtained. Two ensemble means were said to significantly different at the $5 \%$ level if their $95 \%$ confidence intervals did not intersect. (b) Same as (a) except with $\alpha_{\mathrm{c}}=0.01$ and $\alpha_{\mathrm{geo}}=0.01$. All means for the geometric test in panel (b) are significantly different at the $5 \%$ level from the means for the areawise test.

approximately 11 and $N_{\mathrm{c}}(0.18,0.05)=15$ so that $r_{\text {false }}=$ 0.73 and therefore one can expect $36 \%$ more false positives. However, this calculation is an overestimate because the output of the cumulative areawise test is the union of pathway elements as shown in Fig. 9 and discussed in Sect. 7. In fact, an experiment was conducted by generating 1000 wavelet power spectra of red-noise processes with $\rho=0.5$ and lengths equal to 1000 . The ratio $r_{\text {false }}$ for $\alpha_{\max }=0.18$, $\alpha_{\min }=0.02, \Delta \alpha=0.02, \alpha_{\mathrm{c}}=0.05, \alpha_{\text {geo }}=0.05$, and $\alpha=$ 0.05 was computed for each wavelet power spectra. The mean value of $r_{\text {false }}$ was found to be $r_{\text {false }}=0.82$, slightly higher than the theoretical value. The result implies that one 


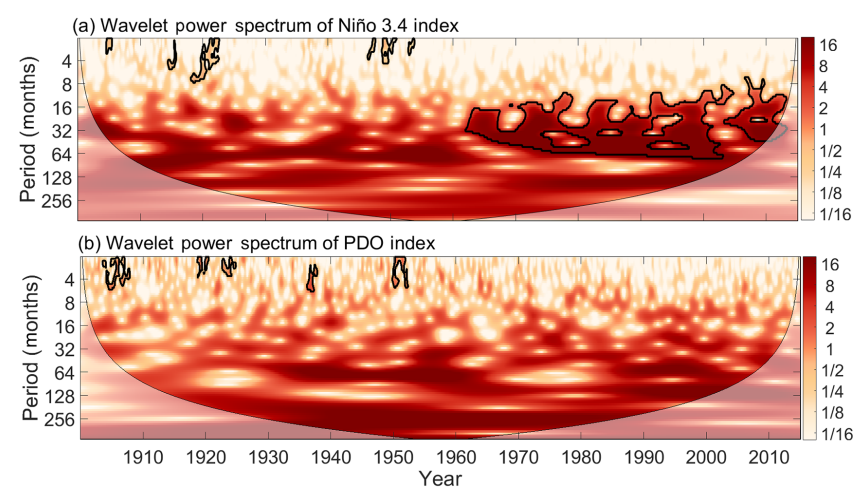

Figure 12. The application of the cumulative areawise test to the (a) Niño 3.4 index and (b) PDO index. Contours enclose regions of $1 \%$ cumulative areawise significance.

can expect the cumulative areawise test to produce $22 \%$ more false positive results. Confidence in results can be enhanced if $\alpha_{\mathrm{c}}=0.01$ without much loss in true positive detection, as shown by the comparison of the curves for $\alpha_{\mathrm{c}}=0.01$ and $\alpha_{\mathrm{c}}=0.05$ in Figs. 11a and b. An experiment similar to the previous experiment with $\alpha_{\mathrm{c}}=0.01$ showed that one can expect $50 \%$ more false positives for the geometric test. The reduction in false positive detection together with relatively high true positive detection suggests that the cumulative areawise test is reliable when $\alpha_{\mathrm{c}}=0.01$.

\section{Climate applications}

The cumulative areawise test was applied to the wavelet power spectra of the PDO and Niño 3.4 indices at the 0.01 level. A red-noise background spectrum was used for each, with $\alpha_{\max }=0.18, \alpha_{\min }=0.02$, and $\Delta \alpha=0.02$.

The wavelet power spectrum for the Niño 3.4 index indicates potential predictive capabilities (Fig. 12a). There is one notable significant feature extending from 1960 to 2014 in the 8-64 month period band. Perhaps just as interesting is the deficit in cumulative areawise significance from 1920 to 1960 in the 8-64 month period band. The deficit could be the result of the 2-7 year mode being modulated by a decadal ENSO mode, a nonlinear paradigm (Timmermann, 2002). Such a modulation would imply that more extreme El Niño phases would be favored if the decadal mode is in a positive regime. On the other hand, results shown in Fig. 12a show that neither the decadal nor the multi-decadal variability exceeded a red-noise background; therefore, modulations would be difficult to predict.

The wavelet power spectrum of the PDO index is shown in Fig. 12b. There is enhanced variance at multi-decadal timescales but the variance does not exceed a red-noise background. Cumulative areawise-significant regions, however, were detected in the 2-8 month period band from 1900 to 1960. The results indicate that the PDO is a red-noise pro- cess, consistent with prior work showing that the PDO results from the oceanic integration of atmospheric white-noise stochastic forcing (Newmann et al., 2003).

\section{Conclusions}

A cumulative areawise test was developed for assessing the significance of features in wavelet power spectra. The test was generally found to have greater statistical power than the geometric test except possibly under high-noise situations, in which case the tests were found to perform similarly. The main advantage of the new testing procedure is that the results are no longer dependent on two significance levels. The geometric test results were found to be very sensitive to the chosen pointwise significance level, making it difficult for researchers to decide what patches are significant and what patches are not significant. The cumulative areawise test was found to detect more true positives relative to the geometric test for some common pointwise and geometric significance levels.

The cumulative areawise test can be applied to wavelet power spectra obtained using other analyzing wavelets such as the Paul and Dog wavelets. Moreover, the results presented for the Morlet wavelet were found to be generally similar to those for the Paul and Dog wavelets. It is recommended, however, that different null distributions be calculated for the different analyzing wavelets.

The cumulative areawise test can also be applied to wavelet coherence, wavelet partial coherence, and multiple wavelet coherence spectra. In these cases, the critical levels of the pointwise test would need to be calculated using Monte Carlo methods. The implementation of the cumulative areawise test, however, is exactly the same as for wavelet power spectra. It is noted that different null distributions for the cumulative areawise test should be used for each, as the cumulative areas of patches in coherence spectra may differ from those found in wavelet power spectra.

The cumulative areawise test applied in this paper was limited to two-dimensional wavelet power spectra. The method, however, may also be applied to global power spectra obtained by time averaging wavelet power at each scale. In this one-dimensional case, geometric pathways would be a nested sequence of arcs. Each member of the nested sequence would be a portion of a global peak that lies above the critical level of the test. Additionally, the one-dimensional test may also prove useful for global coherence (Schulte et al., 2015), which measures the coherence between two time series as a function of wavelet scale. More generally, one can construct an $n$-dimensional cumulative areawise test where the test statistics would be the cumulative sum of $n$-dimensional volumes corresponding to a nested sequence of $n$-dimensional geometric objects.

A potential drawback of the cumulative areawise test is that it may become computationally expensive for very long 
time series. As the length of the time series increases, the number of geometric pathways that need to be calculated also increases. The increase in the number of geometric pathways was found to be nonlinear (not shown), meaning a small change in the time series length yielded a larger change in the number of geometric pathways. Another limitation is that the test relies on the selection of several parameters. One needs to select $I$ and $\Delta \alpha$. Fortunately, the results of procedure were found to change little if $\Delta \alpha$ was smaller than 0.02 .
The results from the climate-mode analysis suggest that the predictability of the PDO is limited and that the multidecadal variability of the PDO is the result of a stochastic process. The Niño 3.4 index, by contrast, was found to have deterministic features, implying that future states of ENSO may be predictable.

A Matlab software package written by the author to implement the cumulative areawise test is available at justinschulte.com. 


\section{Appendix A:}

Let $P_{1}$ and $P_{2}$ be two non-overlapping subsets of a patch $P$ with area $A$ such that $P=P_{1} \cup P_{2}$. Let $A_{1}$ and $A_{2}$ denote the areas of $P_{1}$ and $P_{2}$, respectively. One can thus write

$A=A_{1}+A_{2}$,

$A=r_{1} A+r_{2} A$,

and

$r_{2}=1-r_{1}$,

where $r_{1} r_{2} \in[0,1]$. The centroid of $P$ can be written as

$$
\begin{aligned}
& \frac{1}{A} \iint_{P} s \mathrm{~d} s \mathrm{~d} t=\frac{1}{A} \iint_{P_{1}} s \mathrm{~d} s \mathrm{~d} t+\frac{1}{A} \iint_{P_{2}} s \mathrm{~d} s \mathrm{~d} t, \\
& =\frac{1}{A} \iint_{P} s \mathrm{~d} s \mathrm{~d} t=\frac{r_{1}}{A_{1}} \iint_{P_{1}} s \mathrm{~d} s \mathrm{~d} t+\frac{r_{2}}{A_{2}} \iint_{P_{2}} s \mathrm{~d} s \mathrm{~d} t,
\end{aligned}
$$

or

$C^{\mathrm{s}}=r_{1} C_{1}^{\mathrm{s}}+r_{2} C_{2}^{\mathrm{s}}$,

so that

$$
\frac{C^{\mathrm{s}}-r_{2} C_{2}^{\mathrm{s}}}{r_{1}}=C_{1}^{\mathrm{s}},
$$

where $C_{1}^{\mathrm{s}}$ and $C_{2}^{\mathrm{s}}$ are the scale coordinates of the centroids for $P_{1}$ and $P_{2}$. The equation implies that

$C^{S}-r_{2} C_{2}^{\mathrm{s}}>0$

because $C_{1}^{\mathrm{s}}$ is always positive. The normalized areas of $P$ and $P_{1}$ are given by

$A^{N}=\frac{A}{\left(C^{\mathrm{s}}\right)^{2}}$

and

$A_{1}^{N}=\frac{A_{1}}{\left(C_{1}^{\mathrm{s}}\right)^{2}}$.

Thus,

$r_{\text {norm }}=\frac{A_{1}^{N}}{A^{N}}=\frac{r_{1}^{3}\left(C^{\mathrm{s}}\right)^{2}}{\left(C^{\mathrm{s}}-\left(1-r_{1}\right) C_{2}^{\mathrm{s}}\right)^{2}}$.

At $r_{1}=0, r_{\text {norm }}=0$ because $P_{1}$ has no area. At $r_{1}=1$, $r_{\text {norm }}=1$ because $A_{1}=A$. Moreover, the function is monotonically increasing for $r_{1}=\left[\begin{array}{ll}0 & 1\end{array}\right]$ so that $r_{\text {norm }} \leq 1$. The same arguments hold for $P_{2}$ except that $r_{\text {norm }}$ decreases monotonically. 
Acknowledgements. Support for this research was provided by the National Science Foundation Physical Oceanography Program (award number 0961423) and the Hudson River Foundation (award number GF/02/14). It is a pleasure to thank R. G. Najjar and $\mathrm{N}$. Higson for the helpful advice, which resulted in an improved manuscript.

Edited by: B. Tsurutani

Reviewed by: two anonymous referees

\section{References}

Edelsbrunner, H. and Harer, J.: Persistent homology-a survey, Cotemp. Math., 12, 1-26, 2010.

Efron, B.: Bootstrap methods: another look at the jackknife, Ann. Stat., 7, 1-26, 1979.

Ghrist, R.: Barcodes: the persistent topology of data, B. Am. Math. Soc., 45, 61-75, 2008.

Grinsted, A., Moore, J. C., and Jevrejeva, S.: Application of the cross wavelet transform and wavelet coherence to geophysical time series, Nonlin. Processes Geophys., 11, 561-566, doi:10.5194/npg-11-561-2004, 2004.

Hatcher, A.: Algebraic Topology, Cambridge University Press, New York, 544 pp., 2001.

Higuchi, K., Huang, J., and Shabbar, A.: A wavelet characterization of the North Atlantic Oscillation variation and its relationship to the North Atlantic sea surface temperature, Int. J. Climatol., 19, 1119-1129, 1999.

Labat, D.: Wavelet analysis of the annual discharge records of the world's largest rivers, Adv. Water Resour., 31, 109-117, 2008.

Labat, D.: Cross wavelet analyses of annual continental freshwater discharge and selected climate indices, J. Hydrol., 385, 269-278, 2010.

Lee, Y. J. and Lwiza, K. M. M.: Factors driving bottom salinity variability in the Chesapeake Bay, Cont. Shelf Res., 28, 13521362, 2008.

Lipschutz, S.: Theory and Problems of General Topology. Schaum's Outline, McGraw-Hill, 238 pp., 1965.

Mantua, N. J. and Hare, S. R.: The Pacific Decadal Oscillation, J. Oceanogr., 58, 35-44, 2002.
Maraun, D. and Kurths, J.: Cross wavelet analysis: significance testing and pitfalls, Nonlin. Processes Geophys., 11, 505-514, 2004.

Maraun, D., Kurths, J., and Holschneider, M.: Nonstationary Gaussian processes in wavelet domain: synthesis, estimation, and significance testing, Phys. Rev. E, 75, doi:10.1103/PhysRevE.75.016707, 2007.

Meyers, S. D., Kelly, B. G., and O'Brien, J. J.: An introduction to wavelet analysis in oceanography and meteorology: With application to the dispersion of Yanai waves, Mon. Weather Rev., 121, 2858-2866, 1993.

Newman, M., Compo, G. P., and Alexander, M. A.: ENSO-forced variability of the Pacific Decadal Oscillation, J. Climate, 16, 3853-3857, 2003.

Olsen, J., Anderson, J. N., and Knudsen, M. F.: Variability of the North Atlantic Oscillation over the past 5,200 years, Nature Geosci., 5, 808-812, 2012.

Schulte, J. A., Duffy, C., and Najjar, R. G.: Geometric and topological approaches to significance testing in wavelet analysis, Nonlin. Processes Geophys., 22, 139-156, doi:10.5194/npg-22-1392015, 2015.

Schulte, J. A, Najjar, R. G., and Li, M: Climate mode impacts on streamflow in the mid-Atlantic region of the United States, J. Hydrology: Regional Studies, 5, 80-99, 2016.

Timmermann, A.: Decadal ENSO amplitude modulations: a nonlinear paradigm, Global Planet Change, 37, 135-156, 2003.

Torrence, C. and Compo, G. P.: A practical guide to wavelet analysis, B. Am. Meteor. Soc., 79, 61-78, 1998.

Trenberth, K. E.: The Definition of El Niño, B. Am. Meteor. Soc., 78, 2771-2777, 1997.

Velasco, V. M. and Mendoza, B.: Assessing the relationship between solar activity and some large scale climatic phenomena, Adv. Space Res., 42, 866-878, 2008.

Weerahandi, S.: Exact Statistical Methods for Data Analysis, Springer, 329 pp., 2003.

Whitney, M. M.: A study on river discharge and salinity variability in the Middle Atlantic Bight and Long Island Sound, Cont. Shelf Res., 30, 305-318, 2010.

Wilson, M., Meyers S. D., and Luther, M. E.: Synoptic volumetric variations and flushing of the Tampa Bay estuary, Clim. Dynam., 42, 1587-1594, 2014. 